\title{
IMPACT Recommendations for Improving the Design and Analysis of Clinical Trials in Moderate to Severe Traumatic Brain Injury
}

\author{
Andrew I. R. Maas, ${ }^{*}$ Ewout W. Steyerberg, ${ }^{\dagger}$ Anthony Marmarou, ${ }^{\S}$ Gillian S. McHugh, ${ }^{\text {I }}$ \\ Hester F. Lingsma, ${ }^{\dagger}$ Isabella Butcher, ${ }^{\mathbb{I}}{ }^{2}$ Juan Lu, ${ }^{\S}$ James Weir, ${ }^{\text {II }}$ Bob Roozenbeek, ${ }^{*}{ }^{\dagger}$ and \\ Gordon D. Murray ${ }^{\mathrm{I}}$
}

\begin{abstract}
*Department of Neurosurgery, University Hospital Antwerp, 2650 Edegem, Belgium; ${ }^{\dagger}$ Center for Medical Decision Making, Department of Public Health, Erasmus MC, University Medical Center, 3000 Rotterdam, The Netherlands; ${ }^{\S}$ Department of Neurosurgery, Virginia Commonwealth University Medical Center, Richmond, Virginia 23298; ${ }^{\text {II }}$ Public Health Sciences, Centre for Population Health Sciences, University of Edinburgh Medical School, Edinburgh EH8 9AG, United Kingdom
\end{abstract}

\begin{abstract}
Summary: Clinical trials in traumatic brain injury (TBI) pose complex methodological challenges, largely related to the heterogeneity of the population. The International Mission on Prognosis and Clinical Trial Design in TBI study group has explored approaches for dealing with this heterogeneity with the aim to optimize clinical trials in TBI. Extensive prognostic analyses and simulation studies were conducted on individual patient data from eight trials and three observational studies. Here, we integrate the results of these studies into the International Mission on Prognosis and Clinical Trial Design in TBI recommendations for design and analysis of trials in TBI:
\end{abstract}

- Details of the major baseline prognostic characteristics should be provided in every report on a TBI study; in trials they should be differentiated per treatment group. We also advocate the reporting of the baseline prognostic risk as determined by validated prognostic models.

- Inclusion criteria should be as broad as is compatible with the current understanding of the mechanisms of action of the intervention being evaluated. This will maximize recruitment rates and enhance the generalizability of the results.

- The statistical analysis should incorporate prespecified covariate adjustment to mitigate the effects of the heterogeneity.

- The statistical analysis should use an ordinal approach, based on either sliding dichotomy or proportional odds methodology.

Broad inclusion criteria, prespecified covariate adjustment, and an ordinal analysis will promote an efficient trial, yielding gains in statistical efficiency of more than $40 \%$. This corresponds to being able to detect a $7 \%$ treatment effect with the same number of patients needed to demonstrate a $10 \%$ difference with an unadjusted analysis based on the dichotomized Glasgow outcome scale. Key Words: Traumatic brain injury, clinical trials, study design, sliding dichotomy, covariate adjustment, prognosis.

\section{INTRODUCTION}

Maas et al., ${ }^{1}$ in this issue, provide an up-to-date review of the disappointing history of the clinical testing of interventions for the treatment of acute traumatic brain injury (TBI). In spite of extremely promising pre-clinical data and early phase trials, no agent has yet been shown convincingly in a phase III trial to have clear benefit in
Address correspondence and reprint requests to: Andrew I. R. Maas, Department of Neurosurgery, University Hospital Antwerp, Wilrijkstraat 10, 2650 Edegem, Belgium. E-mail: andrew.maas@uza.be. terms of improving functional outcome after TBI. The reasons for this failure are numerous and have been widely debated. At least part of the explanation comes from limitations in the trial designs and analyses, which have typically been used in TBI. This was the motivation to form a multidisciplinary research group with the goal of developing better approaches to the design and analysis of phase III TBI trials.

\section{The IMPACT project}

The International Mission on Prognosis and Clinical Trial Design in TBI (IMPACT) study group was initiated 
in 2003 with financial support from the U.S. National Institutes of Health. This collaborative venture currently includes clinical, epidemiological and statistical investigators from the University Hospital Antwerp, Belgium, the Erasmus MC, University Medical Center Rotterdam, The Netherlands, the University of Edinburgh, Scotland, and the Medical College of the Virginia Commonwealth University in Richmond, Virginia.

The global aim of the study group was to optimize the design and analysis of clinical trials in TBI to increase the likelihood of demonstrating the benefit of a truly effective new therapy or therapeutic agent in TBI. ${ }^{2}$

The IMPACT investigators were granted access to individual patient data, initially of eight randomized controlled trials and three observational studies, including a total of 9205 patients. Details of the constituent studies and data management of the IMPACT database have been previously described. ${ }^{3}$ Relevant variables from the individual studies were reformatted and merged into a large common dataset forming the basis for exploring concepts to improve the design of clinical trials in TBI.

The focus was on methodological approaches for dealing with the heterogeneity inherent to the TBI population. This heterogeneity relates to clinical severity and baseline prognostic risk. Consequently, a substantial focus of the work was directed toward prognostic analysis prior to exploring methodological approaches to increase statistical power. Identification of robust covariates and the development of prognostic models formed the cornerstone for explorations on how to best deal with the heterogeneity inherent to TBI populations. These explorations involved extensive simulation studies, addressing selection and prognostic targeting on enrollment, covariate adjustment, and ordinal approaches to the analysis. Detailed results of the specific explorations have been reported $^{4-7}$ (McHugh GS, Butcher I, Steyerberg EW, et al. Novel approaches to the analysis of ordinal outcome data in randomized controlled trials in traumatic brain injury: results from the IMPACT project. Clinical Trials. In press.). The aim of this article is to integrate the results of these different studies into general recommendations and to summarize the evidence underpinning the recommendations.

\section{CRITICAL APPRAISAL OF PRECLINICAL AND PHASE II DATA}

Although detailed analysis of preclinical data and of the results of phase II studies was not directly the focus of the IMPACT project, the investigators were struck on a number of occasions by minor and occasionally major deficiencies in the prestudy workup. For example, preclinical studies had sometimes been limited to investigations in a single model or in a single species. Time windows were seldom investigated, especially in the older studies, and uncertainty was often present as to whether the agent under investigation would penetrate sufficiently into brain tissue. Interesting lessons were learned. Only after the Tirilazad studies did it become clear that metabolism in females is different than in males, leading to lower, often subtherapeutic plasma levels. Glutamate excitotoxicity was considered a major mechanism involved in the development of secondary brain damage at the time that many studies were initiated on neuroprotective compounds directed at the N-MethylD-aspartate glutamate receptor antagonist, but later studies showed that glutamate excitotoxicity primarily occurs in patients with contusions or traumatic subarachnoid hemorrhage and may not be so relevant in patients with more diffuse injuries. Critical reappraisal of the phase II study on dexanabinol ${ }^{8}$ showed imbalances between placebo and treatment groups regarding radiological signs of raised intracranial pressure on admission, thus confounding interpretation of the reportedly beneficial effect of Dexanabinol (Pharmos Corp, Iselin, NJ) in controlling raised intracranial pressure. It would appear that investigators, and on occasion, even members of a Steering Committee, may be carried away by the enthusiasm with which pharmaceutical companies sometimes present preclinical or early phase II data. We note a tendency to over-emphasize positive results and have experienced that when we review the raw data, the evidence may be less strong. Preferably, expert reference laboratories should be involved in at least some of the preclinical workup and we recommend even then a careful scrutiny of the raw data rather than relying on what is perhaps selectively presented. This holds in particular when studies have been performed in-house, and not submitted for publication in a peer-reviewed journal. We strongly encourage submission of all results of preclinical work for publication.

\section{PROGNOSTIC ANALYSIS}

- Details of the major baseline prognostic characteristics should be provided in every report on a TBI study; in trials they should be differentiated per treatment group. We also advocate the reporting of the baseline prognostic risk as determined by validated prognostic models.

The motivation for the IMPACT prognostic studies was to use the baseline prognostic risk in the exploration of novel methodological approaches for dealing with the heterogeneity that is typical of TBI trial populations. Hence, we focused on characteristics readily available on admission. Associations between predictors and outcome were explored in univariate analysis, and subsequently the independent predictive value of a measurement determined in multivariable analysis adjusting for the ef- 
fects of other predictors. The primary outcome measure was the 6-month Glasgow Outcome Scale (GOS). Relatively novel statistical approaches for the field of TBI were used toward the prognostic analysis. ${ }^{9}$ Proportional odds methodology was applied to take full advantage of the ordinal nature of the GOS and associations expressed in terms of the odds ratio for a worse versus better outcome. Conceptually this proportional odds ratio is a summary odds ratio over the different splits for collapsing the GOS into a binary variable. Forest plots were used to demonstrate consistency of odds ratios across studies. A random effects model was used to pool these estimates over all studies to give a summary odds ratio per covariate. Spline functions were used to present the association of continuous variables (e.g., age, blood pressure, laboratory data) with outcome in a continuous fashion. The results of this extensive prognostic analysis were reported in a set of articles published in the Journal of Neurotrauma. ${ }^{10-17}$ This work confirmed the predictive value of many known predictors, but is now being analyzed in numbers far greater than previously possible with a much greater precision, and newer predictors, such as race, education, and various laboratory values have been identified. The strength of the effects of the main predictors in moderate and severe TBI are summarized in Table 1.

\section{PROGNOSTIC MODELS}

We successfully developed three prognostic models of increasing complexity, using baseline characteristics available on admission: (1) a core model, containing three well-known predictors (i.e., age, Glasgow Coma Motor Score, and pupillary reactivity); (2) an extended model, including computed tomographic characteristics (CT classification and traumatic subarachnoid hemorrhage) and secondary insults (i.e., hypoxia or hypotension); (3) a laboratory model, including laboratory values on admission (i.e., $\mathrm{Hb}$ and glucose). All three models were developed to predict mortality and unfavorable outcome (GOS score of 1,2, or 3) at 6 months after injury. The models have been published ${ }^{18}$ and are in the public domain (see IMPACT website: www.tbi-impact.org). When developing prognostic models, external validation is essential but has seldom been performed in TBI. ${ }^{19,20}$ For external validation of the IMPACT models, collaboration was sought with the Corticosteroid Randomization After Significant Head injury (CRASH) trial collaborators. The IMPACT models were validated on the severe and moderately injured patients, enrolled into the CRASH trial, and reciprocally models developed by the CRASH trial collaborators (see http:// www.crash2.lshtm.ac.uk/risk\%20calculator/index.html) were validated on the IMPACT database. ${ }^{21}$ Discriminatory performance (i.e., how well patients with different

Table 1. Strength of Prognostic Effect of Main Predictors in Moderate and Severe TBI Grouped By Domain

\begin{tabular}{|c|c|c|c|}
\hline Predictor & Reference Category & Category & $\begin{array}{l}\text { Pooled Common OR } \\
\quad(95 \% \mathrm{CI})\end{array}$ \\
\hline \multicolumn{4}{|l|}{ Demographics } \\
\hline Age & -Continuous & 75 vs 25 th percentile & $2.14(2.00-2.28)$ \\
\hline Clinical severity & -Localizing/obeying commands & -None & $5.30(3.49-8.04)$ \\
\hline \multirow{4}{*}{ GCS motor score } & & -Extension & $7.48(5.60-9.89)$ \\
\hline & & -Abnormal flexion & $3.58(2.71-4.73)$ \\
\hline & & -Normal flexion & $1.74(1.44-2.11)$ \\
\hline & & -Missing/untestable & $2.20(1.66-2.92)$ \\
\hline \multirow{2}{*}{ Pupillary reactivity } & -Both reacting & -One reacting & $2.71(2.36-3.12)$ \\
\hline & & -Both nonreacting & $7.31(5.35-9.99)$ \\
\hline \multicolumn{4}{|l|}{ Second insults } \\
\hline -Hypoxia & -No & -Suspect/definite & $2.08(1.69-2.56)$ \\
\hline -Hypotension & $-\mathrm{No}$ & -Suspect/definite & $2.67(2.09-3.41)$ \\
\hline \multicolumn{4}{|l|}{ Structural damage } \\
\hline \multirow[t]{3}{*}{ CT classification } & -Diffuse injury class II & -Normal & $0.45(0.31-0.67)$ \\
\hline & & -Swelling/shift & $2.62(2.13-3.21)$ \\
\hline & & -Mass lesion & $2.18(1.83-2.61)$ \\
\hline Traumatic subarachnoid hemorrhage & -No & -Yes & $2.64(2.42-2.89)$ \\
\hline Epidural hematoma & $-\mathrm{No}$ & -Yes & $0.64(0.56-0.72)$ \\
\hline \multicolumn{4}{|l|}{ Laboratory values } \\
\hline -Glucose & -Continuous & 75 vs 25 th percentile & $1.68(1.54-1.83)$ \\
\hline -Hemoglobin & -Continuous & 75 vs 25 th percentile & $0.69(0.60-0.78)$ \\
\hline -Prothrombin time & -Continuous & 75 vs 25 th percentile & $1.41(0.99-1.99)$ \\
\hline
\end{tabular}

$\mathrm{CI}=$ confidence interval; $\mathrm{CT}=$ computed tomographic; $\mathrm{GCS}=$ Glasgow coma score; $\mathrm{OR}=$ odds ratio; $\mathrm{TBI}=$ traumatic brain injury. For continuous variables the odds ratios are expressed as the effect of changing from the 25th percentile to the 75 th percentile of the distribution. 
Table 2. Discriminative Properties of IMPACT Models

\begin{tabular}{lccc}
\hline & $\begin{array}{c}\text { Core } \\
\text { Model }\end{array}$ & $\begin{array}{c}\text { Extended } \\
\text { Model }\end{array}$ & $\begin{array}{c}\text { Laboratory } \\
\text { Model }\end{array}$ \\
\hline $\begin{array}{l}\text { Internal validation in } \\
\text { RCTs }\end{array}$ & 0.75 & 0.78 & 0.79 \\
$\begin{array}{l}\text { Internal validation in } \\
\text { observational } \\
\text { studies }\end{array}$ & 0.81 & 0.83 & - \\
External validation & 0.78 & 0.79 & - \\
\hline
\end{tabular}

RCTs $=$ randomized controlled trials.

Discriminative performance is expressed as the area under the receiver operating characteristic curve.

outcomes can be differentiated) was expressed as the area under the receiver operating characteristic curve (AUC). We found an AUC of 0.79 both for the core and extended models, indicating good discrimination. Calibration (i.e., how well-predicted probabilities agree with actually observed frequencies of outcome) was assessed graphically and tested with the Hosmer-Lemeshow goodness-of-fit test. A satisfactory agreement between predicted and observed outcomes was found, especially for the prediction of a 6-month outcome in patients from high-income countries within CRASH (Table 2). The laboratory model could not be validated on the CRASH dataset, as data on laboratory values were lacking.

The development of these prognostic models provided a sound basis for dealing with the heterogeneity of TBI populations, both in the enrollment and in the analysis phase of clinical trials. However, they have much wider applications for clinical practice. Not only can the prognostic risk be determined in individual patients, but TBI populations may be characterized by their prognostic risk distribution. Furthermore, the baseline prognostic risk estimate may be used toward benchmarking the quality of healthcare delivery of TBI. Performance indicators have been developed and tested for many diseases, but not for TBI. Benchmarking is mainly limited to comparing crude mortality rates of specific hospitals against national norms. Standardized mortality rates and calculation of the standardized mortality ratio (observed deaths/expected deaths) have not been developed specifically for TBI. In the epidemiological literature, standardized mortality rates are generally adjusted for age and sex, and in intensive care medicine for baseline characteristics, based on scoring systems such as Apache II, Trauma Injury Severity Score (TRISS), or Simplified Acute Physiology Score (SAPS) II/III. The applicability of these scores for the indication of TBI is doubtful. Moreover, in TBI, functional outcome is much more relevant than mortality. Predictive models provide individual risk estimates and thus can set a baseline for clinical audits and benchmarking by permitting analysis of observed versus expected outcome. As the models have been developed not only for mortality but also for functional outcome (6-month GOS), we consider these models of great potential relevance for assessing the quality of healthcare delivery. We acknowledge that the validity of these models in other settings still needs to be determined, and that epidemiologic patterns and treatment standards change, and also that a regular process of updating the models will be required.

\section{DEALING WITH THE HETEROGENEOUS POPULATIONS IN TBI TRIALS}

- Inclusion criteria should be as broad as is compatible with the current understanding of the mechanisms of action of the intervention being evaluated. This will maximize recruitment rates and enhance the generalizability of the results.

- The statistical analysis should incorporate prespecified covariate adjustment to mitigate the effects of the heterogeneity resulting from the use of broad inclusion criteria.

Sample size calculations traditionally have started from the hypothesis that there will be approximately a $50 / 50$ distribution of unfavorable versus favorable outcome in the placebo population. The aim is to detect an improved favorable outcome by an absolute value of $10 \%$. In the traditional sample size calculation, this will yield an estimated number of approximately 800 patients to detect such a difference with a statistical power of $80 \%$ at the $5 \%$ significance level (two-sided). However, it is seldom realized that the assumption underpinning these calculations is that there is a priori risk of unfavorable outcome of $50 \%$ in every individual patient. When this risk varies substantially, much greater sample sizes are required. The number of patients with an extreme prognosis (i.e., prognostic risk estimate of more than $80 \%$ or less than $20 \%$ for unfavorable outcome) included in the IMPACT database varies between 25 and $45 \%$, depending on the studies selected (randomized controlled trials or surveys) and the models used (Table 3 ). We conclude that the high percentage of patients with an extreme prognosis confounds sample size calculations. If this is ignored, actual statistical power will be substantially lower.

An appealing strategy is to target the study to patients with an intermediate risk estimate (i.e., a risk of 20 to $80 \%$ of unfavorable outcome). Indeed, Machado et al. ${ }^{22}$ demonstrated the validity of this approach in simulation studies and found that statistical power could be increased by prognostic targeting. However, the disadvantage of this approach is a decrease in recruitment leading to a corresponding longer trial duration. We investigated the effects of stricter enrollment criteria on recruitment and found that the application of stricter criteria for 
Table 3. Classification of Patients from Three Surveys and Eight Randomized Controlled Trials Included in the IMPACT Database According to Their Baseline Risk for Unfavorable Outcome

\begin{tabular}{|c|c|c|c|c|}
\hline \multirow[b]{2}{*}{ Models } & \multirow{2}{*}{$\begin{array}{c}\text { Number of Patients } \\
\text { Eligible for Risk } \\
\text { Estimation }\end{array}$} & \multicolumn{3}{|c|}{ Number of Patients per Risk Category (\%) } \\
\hline & & $\begin{array}{l}\text { Low Risk } \\
0 \%-20 \%\end{array}$ & $\begin{array}{c}\text { Intermediate Risk } \\
21 \%-80 \%\end{array}$ & $\begin{array}{c}\text { High Risk } \\
81 \%-100 \%\end{array}$ \\
\hline \multicolumn{5}{|l|}{ Surveys } \\
\hline Core Model* & 2217 & $202(9 \%)$ & $1269(57 \%)$ & $746(34 \%)$ \\
\hline $\begin{array}{l}\text { Extended } \\
\text { Model }^{\dagger}\end{array}$ & 2217 & $247(11 \%)$ & $1208(54 \%)$ & $762(34 \%)$ \\
\hline \multicolumn{5}{|l|}{ RCTs } \\
\hline Core Model* & 6292 & $1040(17 \%)$ & $4733(75 \%)$ & $519(8 \%)$ \\
\hline $\begin{array}{l}\text { Extended } \\
\text { Model }^{\dagger}\end{array}$ & 4782 & $1168(24 \%)$ & $3160(66 \%)$ & $454(10 \%)$ \\
\hline
\end{tabular}

RCTs $=$ randomized controlled trials.

Categories were defined according to the risk of unfavorable outcome, as estimated by the prognostic models as designed by the IMPACT investigators. ${ }^{18}$

Percentages may not add up to $100 \%$ because of rounding. Numbers of patients may differ from numbers reported elsewhere because of differences in recorded variables per study and selection of nonmissing outcome and age $<14$.

*Core model $=$ age, Glasgow Coma Motor Score, and pupillary reactivity.

${ }^{\dagger}$ Extended model = core model, plus hypoxia, hypotension, and computed tomographic characteristics (i.e., Marshall CT classification and traumatic subarachnoid hemorrhage).

pupillary reactivity, time window, motor score, Glasgow Coma Motor Score, and age resulted in a mean reduction of recruitment by $41 \% .^{6}$ Therefore, targeting TBI trials to patients with an intermediate prognostic risk is not efficient, as benefits in terms of statistical power are outweighed by adverse effects on recruitment and trial duration.

An alternative for dealing with prognostic heterogeneity is the application of covariate adjustment in the analysis phase, with the predictors as identified from the prognostic analyses. We found that covariate adjustment yielded a reduction of sample size of more than $25 \%{ }^{4}$ (Table 4). This effect was much greater than previously observed for cardiovascular trials. ${ }^{23}$ Because covariate adjustment had no adverse effects in terms of recruitment, the reduction of required sample size translates to a corresponding reduction in the study duration.

\section{ORDINAL APPROACHES IN THE PRIMARY EFFICACY ANALYSIS}

- The statistical analysis should use an ordinal approach based on either sliding dichotomy or proportional odds methodology. This will yield substantial efficiency gains in comparison with the conventional dichotomous analysis.

Most phase III trials in TBI have used the GOS assessed at 6 months after injury as the primary efficacy endpoint. For analysis, this 5-point ordinal scale is commonly dichotomized into unfavorable (death, vegetative state, or severe disability) versus favorable (moderate disability or good recovery) without relating the point of dichotomization to the initial prognostic risk. Treatment effects are then analyzed versus the resulting binary outcome measure, using either a simple chi-square test or logistic regression incorporating effects of the baseline covariates. Collapsing a continuous or an ordinal measure to a binary scale discards potentially valuable information. ${ }^{24}$ Moreover, as previously discussed, and as illustrated by Machado et al., ${ }^{22}$ patients with an extreme

Table 4. Summary of the Sample Size Reduction Resulting from Ordinal Analysis and/or Covariate Adjustment*

\begin{tabular}{lccc}
\hline & \multicolumn{2}{c}{ Reduction in Sample Size (\%) } \\
\cline { 2 - 4 } Statistical Approach & Median & IQR & Range \\
\hline $\begin{array}{l}\text { Conventional } \\
\text { dichotomy }\end{array}$ & Reference & Reference & Reference \\
$\begin{array}{l}\text { Conventional } \\
\text { dichotomy } \\
\text { covariate }\end{array}$ & 26 & $20-29$ & $14-29$ \\
$\quad \begin{array}{l}\text { adjustment } \\
\text { Proportional odds } \\
\quad \text { analysis }\end{array}$ & 23 & $19-24$ & $41-57$ \\
$\begin{array}{l}\text { Proportional odds } \\
\quad+\text { covariate }\end{array}$ & 49 & $45-53$ & $41-57$ \\
$\begin{array}{l}\text { adjustment } \\
\text { Sliding dichotomy }\end{array}$ & 30 & $29-37$ & $16-45$ \\
$\begin{array}{l}\text { Sliding dichotomy } \\
\quad+\quad \text { covariate }\end{array}$ & 40 & $34-44$ & $25-51$ \\
$\quad$ adjustment & & & \\
\hline
\end{tabular}

$\mathrm{IQR}=$ interquartile range.

These results pertain to those obtained with the use of a 7 covariate model (age, Glasgow Coma Motor Score, pupils, hypoxia, hypotension, computed tomographic classification, and traumatic subarachnoid hemorrhage).

In the sliding dichotomy approach we differentiated 3 bands of equal numbers. 
baseline prognosis have little potential to contribute toward the statistical power of a trial when analyzed using the conventional dichotomy of the GOS. A patient's prognosis can be so good that they will almost inevitably achieve a favorable outcome, even without the benefits of an effective new intervention, or it is so poor that it is extremely unlikely that even an effective intervention would improve their outcome to such an extent that it would move from being unfavorable to favorable. By using the full ordinal scale it becomes possible for a poor prognosis patient whose outcome is improved from vegetative state to severe disability, or a good prognosis patient whose outcome is improved from moderate disability to good recovery to contribute toward demonstrating a beneficial treatment effect.

Therefore it is better to exploit the ordinal nature of the GOS and to relate the outcome to the prognostic risk for individual patients. Two different approaches for this were explored in extensive simulation studies of proportional odds analysis and application of the sliding dichotomy.

In the proportional odds approach, the model considers every possible way in which an ordinal scale can be dichotomized, assuming that the odds ratio for a better outcome versus a worse outcome is identical wherever the scale is dichotomized. In this approach, each patient contributes to the underlying analyses. Hence, we obtain an overall estimate of the shift in outcome across the GOS. From this perspective it has been proposed to refer to the use of the proportional odds model as a "shift analysis." 25

In the sliding dichotomy approach, the point of dichotomy of the GOS is differentiated according to the baseline prognostic risk estimated in individual patients. Thus, for a patient with very severe injuries, survival is relevant, and the split is made between death/vegetative and severe disability, and for patients with less severe injuries, any outcome worse than good recovery may be considered unfavorable, and the split is consequently made between the categories of moderate disability and good recovery. This approach is in contrast to the conventional dichotomous analysis of the GOS, which requires a substantial number of treated patients to cross a prespecified, fixed, and artificially determined boundary, and it ignores all other transitions, such as that from moderate disability to good recovery. This goes against a clinical sense and also lacks statistical sensitivity. The concept of the sliding dichotomy approach has been embraced by the clinical field and has been adopted for the primary analysis of a number of phase III trials in TBI, stroke, and intracerebral hemorrhage. ${ }^{26,27}$ Use of the sliding dichotomy approach requires robust prognostic models to reliably provide a baseline risk estimate in individual patients, which is a prerequisite for defining the appropriate split for dichotomization in individual patients.

Exploring the benefits of ordinal analysis necessitated extensive simulation studies (McHugh et al., submitted for publication). In these simulation studies, the outcome categories death and vegetative state were pooled, both on statistical and ethical grounds. Simulation studies were run across all 11 studies incorporated in the IMPACT database. The main results are summarized in Table 4.

In-depth studies were performed to explore possible consequences of violation of the proportional odds assumption in which the treatment effect is different at the various potential cuts for the GOS. We also explored the use of a three versus a seven predictor model. For the sliding dichotomy, another question was whether to split patient groups into prespecified prognostic categories or divide the population into equal bands, and whether three, four, or five bands or categories would be more appropriate. We found that the proportional odds models gave consistent gains in efficiency, even when the proportional odds assumption was violated (e.g., in the case that a treatment effect only operates on the risk of mortality, and not on the further categories of the GOS). In the use of the sliding dichotomy splitting the population by baseline prognostic risk into three bands of equal numbers gave the most robust results.

These findings demonstrate that substantial efficiency gains can be obtained in phase III trials in TBI by exploiting the ordinal nature of the GOS. The proportional odds models consistently showed slightly greater gains in efficiency compared with the sliding dichotomy approach. However, the sliding dichotomy may be intuitively more attractive to clinicians as it allows a simple exploration of a better than expected outcome at the level of individual patients. The choice between the two ordinal approaches primarily involves a value judgment. The sliding dichotomy sacrifices some of the potential increase in efficiency in favor of transparency and clinical appeal. The proportional odds model is more efficient from a statistical perspective, but perhaps less appealing for a clinical audience. At this point in time, we recommend that both approaches are appropriate for the analysis of TBI trials. The results are convincing, and we strongly believe that the conventional dichotomous analysis should be discarded from the trialist's toolbox.

\section{CONCLUSIONS AND FUTURE DIRECTIONS}

The IMPACT studies have yielded important contributions for advancing the field of clinical research in TBI. The combined analysis of a large series of individual patient data demonstrates the great potential of such studies. In fact, this may be in many cases the best source for evidence concerning many aspects of care, as it will 
be impossible to perform randomized controlled trials for every single treatment approach for which we would like to see more evidence. The difficulties which we experienced in extracting and harmonizing variables across datasets into a common format illustrate the necessity to agree on a common format for selection and coding of variables collected during TBI studies. This experience helped stimulate a multidisciplinary interagency initiative in the United States, providing recommendations for common data elements. We would like to see this initiative evolve as an international effort, which has the potential to set global standards for data collection and coding in TBI. This will constitute an important step to standardize in a way that will allow proper comparisons and permit real progress in designs.

The extensive prognostic analysis has defined and confirmed the predictive value of many known predictors more precisely, it has yielded new predictors, and it has resulted in the presentation of validated prognostic models for use in moderate and severe TBI. These models have much wider applications than in only the context of a trial design. We recognize that these models were developed, at least in part, on older TBI series, and that epidemiology, trauma organization, and approaches to treatment may vary between settings and change in time. The external validation on the CRASH trial, and ongoing, preliminary validation on more recent TBI series, however, demonstrate their applicability across different settings and their relevance to current day practice. Nevertheless, we recommend a process of continuous updating of the models, and furthermore, extensive assessment of their validity in other settings. ${ }^{28}$

The explorations for improving the design and analysis of clinical trials in TBI illustrate how crude our approaches over the past decades have really been and that we may not have been giving new therapies a fair chance. The recommendation for relatively broad inclusion criteria, performing prespecified covariate adjustment in the analysis phase, constitutes a clear change of direction from current approaches in which studies tend to use strict enrolment criteria in an attempt to decrease the inherent heterogeneity of the population. The potential benefit (over 40\%) in terms of statistical power when prespecified covariate adjustment is combined with an ordinal approach to outcome analysis is impressive and should become the standard for the future. It should be recognized however that although these recommendations may partly solve the problem of heterogeneity in terms of injury severity and prognostic risk, they do not address heterogeneity related to mechanism. Pathophysiologic mechanisms are different in patients with for example contusions (large inflammatory component) compared to patients with diffuse axonal injury (less inflammation, more calcium mediated damage). The concept of mechanistic targeting - the ideal for clinical trials - will require reliable identification of occurrence and time course of pathophysiologic mechanisms in individual patients. Here we see a great challenge for basic scientists and clinical researchers. ${ }^{29}$

The IMPACT studies are continuing; we are including new datasets, permitting critical assessment and validation of findings in more contemporary series. A major focus of the current research concerns the confounding influence of center effects and variations in treatment approaches in multicenter studies. Uncertainty further exists whether the recommendations presented would be equally valid in a large mega trial as in smaller, more focused series. These aspects are currently being investigated in collaboration with the CRASH trial and Trauma Audit and Research Network (TARN) investigators.

A particular focus is also being made on improving approaches to outcome assessment. The GOS is currently accepted by investigators and regulatory authorities alike as the standard for a primary efficacy endpoint. However, misclassification in outcome assignment may occur, and the GOS has been criticized as being relatively insensitive by not capturing improvements in important domains such as cognitive function. Choi et al. ${ }^{30}$ showed that misclassifications on the GOS might not only reduce the overall power of a trial, but also reduce the apparent size of any benefit. This finding was further demonstrated in the simulation study by Lu et al. ${ }^{7}$ in which a $20 \%$ random misclassification on a dichotomous GOS outcome could reduce the treatment effect from the expected 10 to $6.8 \%$ while maintaining the statistical power as a fixed factor. These studies further showed that the treatment arm is likely to be affected more by the misclassification than the placebo arm. Whether these observations are specific to the dichotomous approach or also relevant to ordinal approaches to outcome analysis is currently under investigation. Nevertheless, we recommend implementing efforts to reduce the risk for misclassification. Training of study personnel improves consistency in outcome assignment, ${ }^{31}$ even in experienced centers, and it is recommended.

Outcome after TBI is by definition multidimensional, including neurophysical disabilities, disturbances in mental functioning (e.g., cognitive and executive functioning), and consequential problems in social reintegration. We see a need to explore the feasibility of developing a multidimensional approach to outcome assessment and classification.

Despite the substantial advances resulting from the IMPACT project, many challenges remain. Perhaps these may best be addressed according to the concept of an "open source format" with multidisciplinary and international collaboration, and with investigators and researchers willing to share data and results as these evolve. This direction was already initiated when the 
original stakeholders granted the IMPACT investigators permission to access the individual patient data of their studies for which the IMPACT investigators are deeply grateful.

Acknowledgments: This work was supported by the U.S. National Institutes of Health (Clinical Trial Design and Analysis in TBI Project No. R01 NS-042691).

\section{REFERENCES}

1. Maas AIR, Roozenbeek B, Manley GT. Clinical trials in traumatic brain injury: past experience and current development. Neurotherapeutics 2010;7:115-126.

2. Maas AIR, Marmarou A, Murray GD, Teasdale GM, Steyerberg EW. Prognosis and clinical trial design in traumatic brain injury: The IMPACT Study. J Neurotrauma 2007;24:232-238.

3. Marmarou A, Lu J, Butcher I, et al. IMPACT database of traumatic brain injury: design and description. J Neurotrauma 2007;24:239250.

4. Hernandez AV, Steyerberg EW, Butcher I, et al. Adjustment for strong predictors of outcome in traumatic brain injury trials: $25 \%$ reduction in sample size requirements in the IMPACT Study. J Neurotrauma 2006;23:1295-1303.

5. Murray GD, Barer D, Choi S, et al. Design and analysis of phase III trials with ordered outcome scales-the concept of the sliding dichotomy. J Neurotrauma 2005;22:511-517.

6. Roozenbeek B, Maas AI, Lingsma HF, et al. Baseline characteristics and statistical power in randomized controlled trials: selection, prognostic targeting, or covariate adjustment? Crit Care Med 2009;37:2683-2690.

7. Lu J, Murray GD, Steyerberg EW, et al. Effects of glasgow outcome scale misclassification on traumatic brain injury clinical trials. J Neurotrauma 2008;25:641-651.

8. Knoller N, Levi L, Shoshan I, et al. Dexanabinol (HU-211) in the treatment of severe closed head injury: a randomized, placebocontrolled, phase II clinical trial. Crit Care Med 2002;30:548-554.

9. McHugh GS, Butcher I, Steyerberg EW, et al. Statistical approaches to the univariate prognostic analysis of the IMPACT database on traumatic brain injury. J Neurotrauma 2007;24:251258.

10. Mushkudiani NA, Engel DC, Steyerberg EW, et al. Prognostic value of demographic characteristics in traumatic brain injury: results from the IMPACT study. J Neurotrauma 2007;24:259269.

11. Marmarou A, Lu J, Butcher I, et al. Prognostic value of the Glasgow coma scale and pupil reactivity in traumatic brain injury assessed pre-hospital and on enrollment: an IMPACT analysis. J Neurotrauma 2007;24:270-280.

12. Butcher I, Maas AI, Lu J, et al. Prognostic value of admission blood pressure in traumatic brain injury: results from the IMPACT study. J Neurotrauma 2007;24:294-302.

13. McHugh GS, Engel DC, Butcher I, et al. Prognostic value of secondary insults in traumatic brain injury: results from the IMPACT study. J Neurotrauma 2007;24:287-293.

14. Butcher I, McHugh GS, Lu J, et al. Prognostic value of cause of injury in traumatic brain injury: results from the IMPACT study. J Neurotrauma 2007;24:281-286.
15. Maas AI, Steyerberg EW, Butcher I, et al. Prognostic value of computerized tomography scan characteristics in traumatic brain injury: results from the IMPACT study. J Neurotrauma 2007;24: 303-314.

16. Van Beek JG, Mushkudiani NA, Steyerberg EW, et al. Prognostic value of admission laboratory parameters in traumatic brain injury: results from the IMPACT study. J Neurotrauma 2007;24:315-328.

17. Murray GD, Butcher I, McHugh GS, et al. Multivariable prognostic analysis in traumatic brain injury: results from the IMPACT Study. J Neurotrauma 2007;24:329-337.

18. Steyerberg EW, Mushkudiani N, Perel P, et al. Predicting outcome after traumatic brain injury: development and international validation of prognostic scores based on admission characteristics. PLoS Med 2008;5:e165.

19. Perel P, Edwards P, Wentz R, Roberts I. Systematic review of prognostic models in traumatic brain injury. BMC Med Inform Decis Mak 2006;6:38.

20. Mushkudiani NA, Hukkelhoven CW, Hernandez AV, et al. A systematic review finds methodological improvements necessary for prognostic models in determining traumatic brain injury outcomes. J Clin Epidemiol 2008;61:331-343.

21. MRC CRASH Trial Collaborators, Perel P, Arango M, Clayton T, et al. Predicting outcome after traumatic brain injury: practical prognostic models based on large cohort of international patients. BMJ 2008;336:425-429.

22. Machado SG, Murray GD, Teasdale GM. Evaluation of designs for clinical trials of neuroprotective agents in head injury. J Neurotrauma 1999;16:1131-1138.

23. Steyerberg EW, Bossuyt PM, Lee KL. Clinical trials in acute myocardial infarction: should we adjust for baseline characteristics? Am Heart J 2000;139:745-751.

24. Altman DG, Royston P. The cost of dichotomising continuous variables. BMJ 2006;332:1080.

25. Saver JL. Novel end point analytical techniques and interpreting shifts across the entire range of outcome scales in acute stroke trials. Stroke 2007;38:3055-3062.

26. Mendelow AD, Gregson BA, Fernandes HM, et al. for the STICH investigators. Early surgery versus initial conservative treatment in patients with spontaneous supratentorial intracerebral haematomas in the International Surgical Trial in Intracerebral Haemorrhage (STICH): a randomised trial. Lancet 2005;365:387-397.

27. Maas AI, Murray G, Henney H III, et al. Efficacy and safety of dexanabinol in severe traumatic brain injury: results of a phase III randomised, placebo-controlled, clinical trial. Lancet Neurology 2006;5:38-45.

28. Steyerberg EW, Borsboom GJ, van Houwelingen HC, Eijkemans MJ, Habbema JD. Validation and updating of predictive logistic regression models: a study on sample size and shrinkage. Stat Med 2004;23:2567-2586.

29. Saatman KE, Duhaime AC, Bullock R, Maas AI, Valadka A, Manley GT. Classification of traumatic brain injury for targeted therapies. J Neurotrauma 2008;25:719-738.

30. Choi SC, Clifton GL, Marmarou A, Miller ER. Misclassification and treatment effect on primary outcome measures in clinical trials of severe neurotrauma. J Neurotrauma 2002;19:17-22.

31. Wilson JT, Slieker FJ, Legrand V, Murray G, Stocchetti N, Maas AI. Observer variation in the assessment of outcome in traumatic brain injury: experience from a multicenter, international randomized clinical trial. Neurosurgery 2007;61:123-129. 